Nervenarzt 2012 $\cdot 83: 751-758$

DOI 10.1007/s00115-011-3424-2

Online publiziert: 5. Januar 2012

(c) Springer-Verlag 2012
E. Archavlis ${ }^{1}$ M.C. Nievas ${ }^{2}$

${ }^{1}$ Neurochirurgische Klinik und Ambulanz, Klinikum Offenbach, Akademisches

Lehrkrankenhaus der JWG-Universität Frankfurt am Main, Offenbach

${ }^{2}$ Neurochirurgische Klinik, Städtische Kliniken Frankfurt am Main Höchst, Frankfurt am Main

\title{
Kranioplastik nach supratentorieller dekompressiver Kraniektomie
} Wann ist der Zeitpunkt optimal?

\section{Die Kranioplastik dient gemäß der Definition von Sanan und Haines der Deckung von Kraniektomiedefekten oder der Behebung einer Entstellung des Kraniums [26]. Die dekompres- sive Kraniektomie wird zunehmend bei der Behandlung von erhöhtem Hirndruck infolge eines Schädel-Hirn- Traumas [6, 15], eines ischämischen Schlaganfalls [14, 17, 36], einer Sub- arachnoidalblutung [32] oder einer intrazerebralen Infektion [1] einge- setzt und nach den neuesten kont- rolliert randomisierten Studien führt dieses Verfahren zur Verbesserung der Überlebensrate $[17,36]$ und der Lebensqualität [14] im Vergleich zur konservativen Behandlung.}

Aufgrund der zunehmenden Verbreitung der dekompressiven Kraniektomie [14, 15, 17] häufen sich in den letzten Jahren die Berichte über mittelfristige Folgen auf die Liquorhydrodynamik $[8,12,29]$, die kortikale Hirnperfusion $[34,25,27]$ und den Metabolismus[18, 37], die einen negativen Einfluss auf die neurologische Erholung der Patienten ausüben können [4, 12, 29, 31]. Hinzu kommt das Auftreten unterschiedlicher, oft positions- und mobilisationsdauerabhängiger fluktuierender neurologischer Symptome in zeitlicher Assoziation mit dem Einsinken des Hautlappens, das in der Literatur als „syndrome of the trephined" bezeichnet wird $[8,28,37]$.
Obwohl bei der Indikationsstellung für die supratentorielle dekompressive Kraniektomie klare Kriterien erarbeitet wurden $[6,14,15,17,33,36]$, existieren heute noch keine Leitlinien bezüglich der Deckung von Kraniektomiedefekten. Die aktuelle Zeitspanne zwischen dekompressiver Kraniektomie und Kranioplastik schwankt zwischen 3 und 6 Monaten [30]. In Zentren mit eigener Knochenbank wird häufig der tiefgefrorene Eigenknochen zu Zwecken der Kranioplastik verwendet. Es ist leider wissenschaftlich noch nicht geklärt, wie lange nach dem Ersteingriff dies mit vertretbarem Risiko für Infektion oder Demineralisation geschehen kann [24], ebenso wenig, welche Vorteile oder Nachteile die Anwendung anderer Implantatmaterialien für den Patienten haben kann [38].

Diese Studie führt eine retrospektive Vergleichsanalyse unserer Patienten sowohl für die gebräuchlichen traditionellen Verfahren als auch für die neuen Verfahren im Hinblick auf die Aufklärung aller dieser noch bestehenden Fragen durch.

\section{Patienten und Methoden}

Insgesamt 242 Patienten, die in den Jahren 2001 bis 2007 in der Neurochirurgischen Klinik Frankfurt Höchst einer Kranioplastik nach dekompressiver Kraniektomie unterzogen worden sind, wurden in die vorliegende Studie eingeschlossen (• Tab. 1).
Die supratentorielle dekompressive Kraniektomie wurde bei allen Patienten zur Behandlung eines therapieresistenten erhöhten intrakraniellen Druckes in der Folge verschiedener Grunderkrankungen durchgeführt. $\mathrm{Zu}$ den Ursachen der Kraniektomie zählten bei 85 Patienten eine Subarachnoidalblutung (SAB, alle Patienten mit SAB nach der Hunt-undHess-Klassifikation Grad 3 und 4), bei 59 Patienten ein Trauma, bei 44 Patienten ein Infarkt, bei 30 Patienten eine intrazerebrale Blutung (ICB), bei 8 Patienten eine Infektion, bei 4 Patienten ein Tumor und bei 12 Patienten lagen sonstige Ursachen vor. Nach Entfernung eines Knochendeckels von mindestens $12 \mathrm{~cm}$ im Durchmesser wurde in allen Fällen eine Duraplastik mit synthetischem Material (Tutopach ${ }^{\circ}$, Fa. Tutogen Medical, Neunkirchen) durchgeführt. In 217 (90\%) der 242 Fälle wurde der entfernte autologe Schädelknochen bei -70 C in unserer Knochenbank gelagert und zu einem späteren Zeitpunkt reimplantiert. Bei 10 (4\%) Patienten wurde eine PMMA (Polymethylenmethacrylat)-Plastik durchgeführt. In 4 (2\%) Fällen wurde der autologe Knochen nach Anwendung des Tutoplastverfahrens (Reinigung, Konservierung und Sterilisation) reimplantiert. In 6 (2\%) Fällen wurde ein Glaskeramik Implantat, in 4 (2\%) Fällen ein PEEK (Polyetheretherketone)-Implantat nach vorheriger Planungs-CCT (zerebrale Computertomographie) verwendet und in einem 


\begin{tabular}{|c|c|c|c|c|}
\hline & Gruppe 1 & Gruppe 2 & Gruppe 3 & $\mathbf{p}$ \\
\hline & $n=96$ & $\mathbf{n}=\mathbf{7 7}$ & $n=68$ & \\
\hline Alter (Jahre), MW \pm SD & $53,3 \pm 8,2$ & $51,4 \pm 18,5$ & $52,4 \pm 15,5$ & $>0,5$ \\
\hline Geschlecht (weiblich) & $41(42,7 \%)$ & $34(44,1 \%)$ & $36(52,9 \%)$ & $>0,5$ \\
\hline Standard-Follow-up [Monate (Spanne)] & $43,2(11-49)$ & $42,9(12-52)$ & $43,6(9-62)$ & $>0,5$ \\
\hline Präoperativer GCS-Score, MW & 13 & 13 & 13 & $>0,5$ \\
\hline Zeitpunkt der Kranioplastik (Monate), MW & 5 & 9 & 22 & \\
\hline Ursache der Kraniektomie (\%) & & & & $>0,05$ \\
\hline Trauma & 27 & 22 & 23 & \\
\hline Infarkt & 8 & 17 & 32 & \\
\hline Subarachnoidalblutung & 40 & 35 & 26 & \\
\hline Intrazerebrale Blutung & 17 & 18 & 3 & \\
\hline Infektion & 0 & 0 & 12 & \\
\hline Tumor & 2 & 3 & 0 & \\
\hline Sonstige & 6 & 5 & 3 & \\
\hline Art der Kranioplastik (\%) & & & & $>0,05$ \\
\hline Eigenknochen & 93,7 & 100 & 69 & \\
\hline PMMA & 6,3 & 0 & 5,9 & \\
\hline Tutoplast & 0 & 0 & 7,3 & \\
\hline Glaskeramik & 0 & 0 & 8,8 & \\
\hline PEEK & 0 & 0 & 8,8 & \\
\hline
\end{tabular}

Fall wurde ein Titanimplantat als maßgeschneiderte Plastik nach vorheriger Planungs-CCT implantiert (• Abb. 1). Die Fixation der Implantate erfolgte immer mit Titaniummikroplatten.

Im Anschluss an die Aktenauswertung wurde das neurologische Outcome der Patienten anhand der Glasgow Outcome Scale (GOS) nach der Kranioplastik (vor Entlassung aus dem stationären Aufenthalt) und zu einem späteren Zeitpunkt mittels telefonischer Befragung beurteilt. Bei der GOS werden eine gute Erholung bei minimaler Behinderung mit 5 Punkten und der Tod des Patienten mit einem Punkt bewertet.

Um bestimmte Vorteile der frühen oder späteren Kranioplastik auf die verglichenen Parameter besser zu beurteilen, wurde der Zeitpunkt der Kranioplastik in 3 Gruppen unterteilt:

- Gruppe 1 (ultrafrühe Kranioplastik): o bis inklusive 6. Woche,

- Gruppe 2 (frühe Kranioplastik): 7. bis inklusive 12. Woche und

- Gruppe 3 (späte Kranioplastik): nach 13. Woche.

Die Gruppe der 242 untersuchten Patienten bestand aus 112 Frauen und 130 Männern mit einem Durchschnittsalter von 52,6 Jahren (1-79 Jahre). Bei 234 Patienten war eine unilaterale und bei 8 Patienten eine bilaterale frontotemporoparietale dekompressive Kraniektomie initial durchgeführt worden.

Das Zeitintervall zwischen der Kranioplastik und der telefonischen Befragung lag im Durchschnitt bei 43,4 Monaten. Drei Patienten konnten für die telefonische Befragung nicht erreicht werden und wurden bei der statistischen Aufarbeitung der Daten nicht berücksichtigt.

\section{Gruppeneinteilung nach Zeitpunkt der Kranioplastik}

In der Gruppe 1 (ultrafrühe Kranioplastik) war ein Schädel-Hirn-Trauma (SHT) in 26 Fällen $(27 \%)$, in 8 Fällen (8\%) ein Infarkt, in 38 Fällen (40\%) eine SAB und in 16 Fällen (17\%) eine ICB der Grund für die dekompressive Kraniektomie. In der Gruppe 2 (frühe Kranioplastik) lag in 27 Fällen (35\%) eine SAB, in 13 Fällen (17\%) ein Infarkt, in 17 Fällen (22\%) eine SHT, in 14 Fällen (18\%) eine ICB und in 13 Fällen (18\%) ein Infarkt als Grund für die dekompressive Kraniektomie vor. In der Gruppe 3 war in 8 Fällen (12\%) eine Infektion, in 18 Fällen (26\%) eine SAB, in 22 Fällen (32\%) ein Infarkt, in 16 Fällen $(23 \%)$ eine SHT und in 2 Fällen (3\%) eine ICB ursächlich für die dekompressive Kraniektomie (• Tab. 1). Diese Unterschiede stellten sich im Fisher-Yates-Test als nichtsignifikant dar ( $p>0,05)$.

In Abhängigkeit von den verschiedenen Ursachen der Kraniektomie erfolgte die Kranioplastik jedoch zu unterschiedlichen Zeitpunkten: bei 60\% der kraniektomierten Patienten nach SAB, Trauma und ICB um die 7. Woche, bei Patienten nach Hirninfarkten zu einem späteren Zeitpunkt. Hier hatten $60 \%$ der Patienten erst in der 12. Woche nach Kraniektomie eine Kranioplastik erhalten. Bei kraniektomierten Patienten nach intrazerebralen Infektionen war der Kranioplastikzeitpunkt signifikant noch später angesetzt worden (• Abb. 2).

\section{Outcome}

Der Vergleich des präoperativen neurologischen Zustandes der Patienten in den drei Gruppen ergab vor der Kranioplastik keine wesentlichen Unterschiede (- Abb. 3a). Allerdings wurde eine Besserung des funktionellen und kognitiven Outcomes nach der Kranioplastik bei $88,5 \%$ der Patienten (85 Fälle) in der Gruppe 1 beobachtet. In dieser Gruppe blieb nur bei 6,25\% (11 Fälle) der Patienten der kognitive und funktionelle Status unverändert. In der Gruppe 2 zeigten 63,6\% der Patienten (49 Fälle) eine Besserung des kognitiven und funktionellen Outcomes und 23,4\% der Patienten (18 Fälle) waren unverändert. Nur bei $21 \%$ der Pa- 
tienten (14 Fälle) in der Gruppe 3 wurde eine signifikante funktionelle und kognitive Verbesserung dokumentiert. Bei 76,5\% der Patienten (52 Fälle) blieb der funktionelle und kognitive Zustand der Patienten unverändert. Die neurologische Besserung im Rahmen der GOS wurde in 81 von insgesamt 96 Fällen $(84,4 \%)$ in der Gruppe 1, in 34 von insgesamt 77 Fällen $(44,1 \%)$ in der Gruppe 2 und nur in 9 von insgesamt 68 Fällen $(13,2 \%)$ in der Gruppe 3 festgestellt.

Diese Daten brachten nach dem Fischer-Yates-Test einen signifikanten Unterschied ( $\mathrm{p}=0,0001 ;(\bullet$ Abb. 3b).

\section{Kraniektomiedefekte}

Der kranielle Defekt wurde nach Beurteilung des CCT vor der Kranioplastik in 3 Typen unterteilt:

- Typ 1: schlaffe, konkave, eingesunkene Kraniektomiestelle,

- Typ 2: weiche, leicht konvexe, auf dem Niveau des Kalottenrandes befindliche Kraniektomiestelle,

- Typ 3: konvexe, über dem Niveau des Kalottenrandes befindliche, eventuell auch prall gespannte, hernierte Kraniektomiestelle.

Insgesamt 93 Patienten wurden als Typ 1 eingestuft, 77 Patienten als Typ 2 und weitere 70 Patienten als Typ 3 (• Abb. 4). Die Möglichkeit der kombinierten Versorgung mit Ableitung eines vorhandenen Hydrozephalus und Kranioplastik wird in der Diskussion ausführlich erläutert.

\section{„Syndrom of the trephined"}

Nur 27 der insgesamt 242 Patienten hatten vor der Kranioplastik unter dem sog. „syndrome of the trephined“ gelitten (- Abb. 5). Folgende Symptome waren bei fast allen dieser Patienten vorhanden: Kopfschmerzen, Schwindel, psychiatrische Veränderungen und Unbehagen. In einem Fall wurden fokale Anfälle beobachtet und in 2 weiteren Fällen wurde eine Hemisymptomatik ohne radiologisches Korrelat festgestellt. Diese Symptome waren mobilisationsabhängig, fluktuierend und in zeitlicher Assoziation mit dem Einsinken des Hautlappens.

Nervenarzt 2012 $\cdot 83: 751-758 \quad$ DOI 10.1007/s00115-011-3424-2

C) Springer-Verlag 2012

\section{E. Archavlis · M.C. Nievas}

\section{Kranioplastik nach supratentorieller dekompressiver Kraniektomie. Wann ist der Zeitpunkt optimal?}

\section{Zusammenfassung}

Hintergrund. Ziel dieser Arbeit war es, die bisherigen Erfahrungen der Kalottenplastik im Hinblick auf die verschiedenen Zeitpunkte und Materialien der Kranioplastik zu untersuchen und unter klinischen Aspekten zu bewerten.

Patienten und Methoden. Die Daten von insgesamt 242 Patienten, die einer Kranioplastik unterzogen worden waren, wurden retrospektiv analysiert. Um Aufschluss über das postoperative, funktionelle und kosmetische Ergebnis zu erhalten, wurde im Anschluss an die Aktenauswertung bei diesen Patienten eine telefonische Befragung durchgeführt. Die Patienten wurden gemäß dem Zeitpunkt der Kranioplastik in 3 Gruppen aufgeteilt: ultrafrühe (bis 6. Woche), frühe (6. bis 12 . Woche) und späte Kranioplastik (nach der 13. Woche).

\begin{abstract}
Ergebnisse. Die Analyse der Patientengruppen ergab, dass die ultrafrühe Kranioplastik bei Patienten mit großen Defekten nach dekompressiver Kraniektomie ein besseres Outcome im langfristigen Follow-up hat. Diese Patienten hatten keine gesteigerte Infektions- oder andere Komplikationsraten.

Schlussfolgerung. Die frühe Kranioplastik mittels Reimplantation des Eigenknochens kann bei ausgewählten Patienten als ein sicheres und hilfreiches Verfahren für die schnellere Rehabilitation und Besserung der neurologischen Funktion und Prognose bewertet werden.
\end{abstract}

\section{Schlüisselwörter \\ Schädelknochendefekt · Dekompressive Kraniektomie - Kranioplastik . \\ Reimplantation · Operationszeitpunkt}

\section{Cranioplasty after supratentorial decompressive craniectomy. When is the optimal timing}

\section{Summary}

Background. The purpose of this study was to evaluate the outcome and complication rates of different cranioplastic procedures. Patients and methods. This study retrospectively reviewed 242 consecutive patients who underwent cranioplasty. The indications for craniectomy, the timing and materials employed for the cranial repair procedures were analysed in all patients as well as their early and long-term results. The immediate patient's outcome after cranioplasty was assessed by reviewing medical records and the late outcome was evaluated with a telephone questionnaire. The patients were divided into three groups depending on the timing of cranioplasty [ultra early group (until 6 weeks), early group (7-12 weeks) and delayed group (after 13 weeks following craniectomy)].
Results. The ultra early cranioplasty in the form of reimplantation of the patient's own skull flap led to a rapid improvement of the patient's neurological function and late outcome. The analysis of the registered postoperative complications revealed that there were no significant differences between the groups examined.

Conclusion. This study found that ultra early cranioplasty by reimplanting the patient's own previously removed and frozen skull bone was a safe and successful strategy.

\section{Keywords}

Skull defect · Decompressive craniectomy . Cranioplasty · Reimplantation · Timing
Bei 8 von 10 Patienten in der Gruppe der ultrafrühen Kranioplastik, bei 7 von 8 Patienten in der Gruppe der frühen und bei nur 2 von 9 Patienten in der Gruppe der späteren Kranioplastik fand sich eine Besserung dieser Symptome nach Deckung des Schädeldefektes A B.

\section{Knochenresorption}

Es fand sich kein Zusammenhang zwischen Resorption des Eigenknochens nach Reimplantation und Zeitdauer bis zur Kranioplastik. Insgesamt wurden nur 3 Fälle mit einer Resorption des autologen Implantates festgestellt. Diese Patienten zeigten im CCT das typische Bild mit 

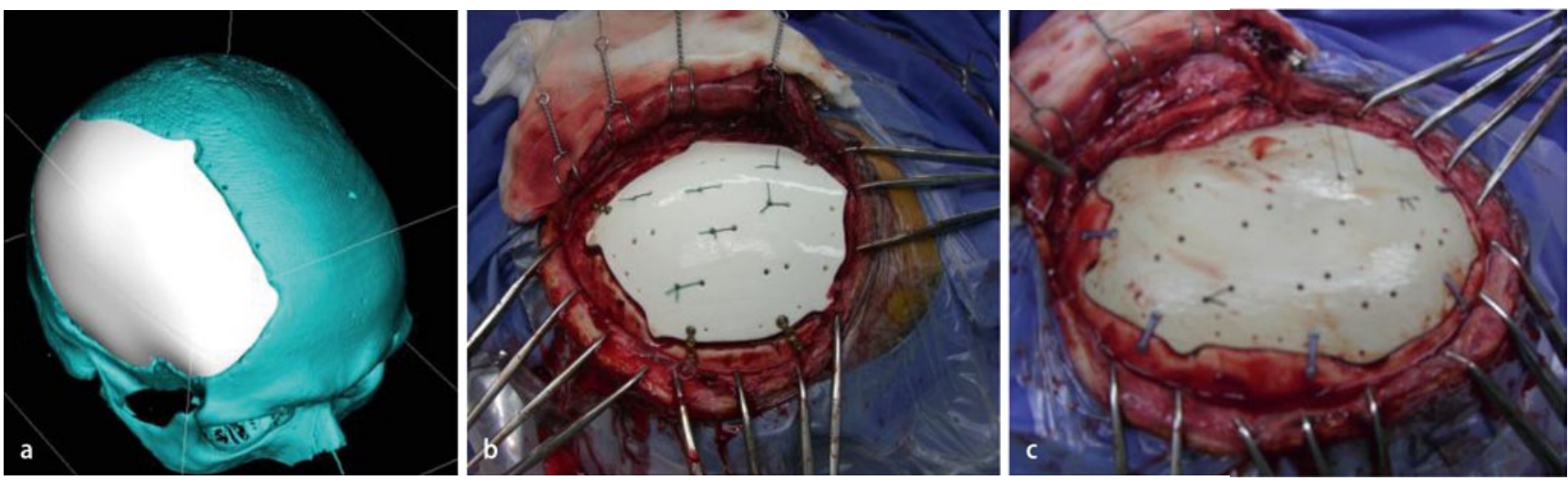

Abb. $1 \Delta$ Implantatmaterialien als Knochenersatzplastik (Keramik, PEEK). a Dreidimensionale CT-Rekonstruktion für ein CADCAM-Implantat. b Intraoperativer Befund bei dem Einbau des Glaskeramik-Bioverit ${ }^{\oplus}$-Implantates. $\mathbf{c}$ Intraoperative Sicht auf ein PEEK-Implantat mit vorgefertigten Löchern für die Anlage von Dura-Hochnähten und für die Fixierung mittels Titanplättchen

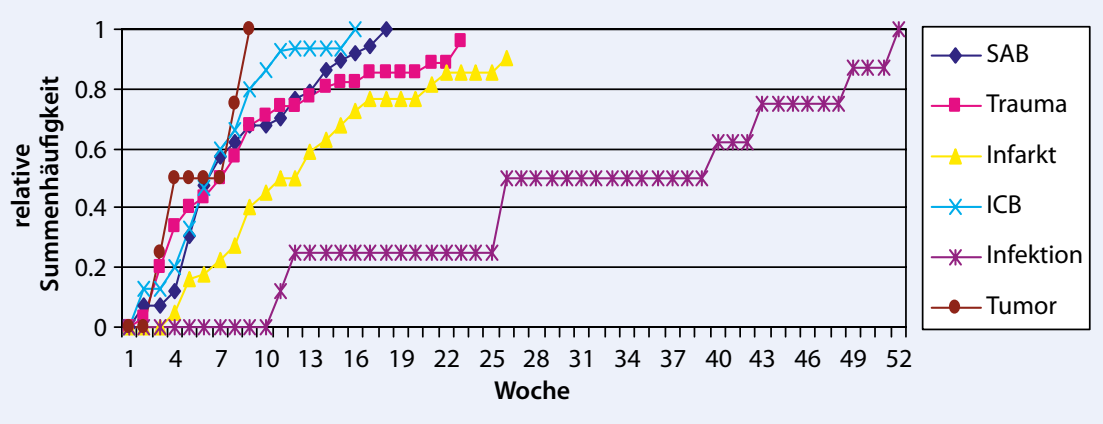

Abb. $2 \Delta$ Kranioplastikzeitpunkt in Abhängigkeit von der Ursache der Kraniektomie. ICB intrazerebrale Blutung, SAB Subarachnoidalblutung

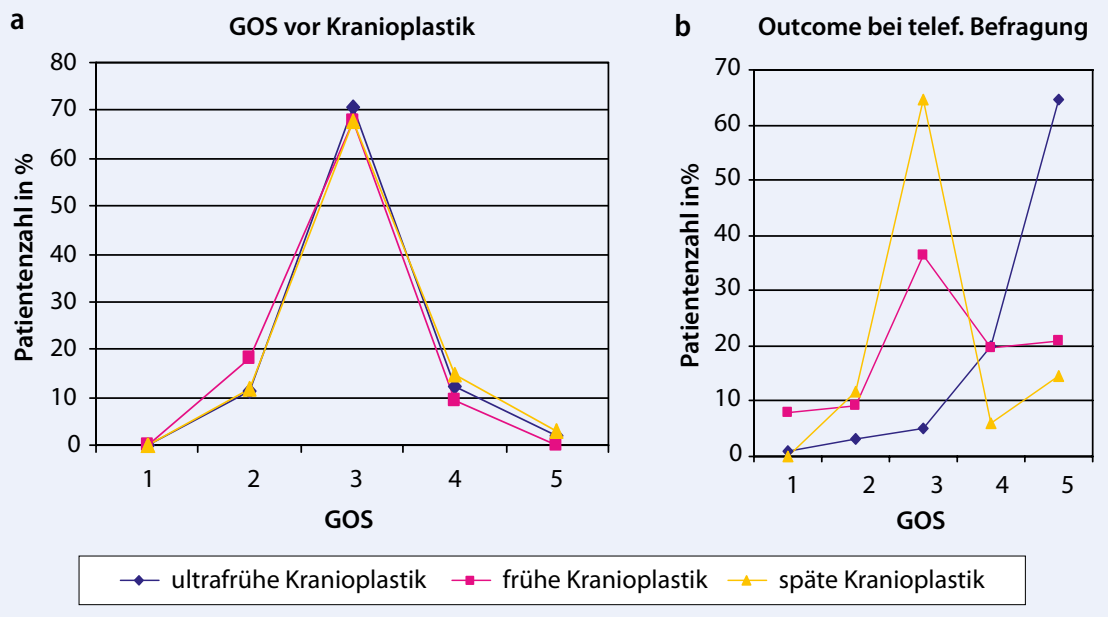

Abb. $3 \Delta$ Glasgow Outcome Scale (GOS) a vor und b nach Kraniektomie

osteolytischen Herden und Ausdünnung des Implantates (• Abb. 6).

\section{Infektiöse Komplikationen}

Alle infektiösen Komplikationen wurden in Bezug auf folgende Parameter analy- mit der Folge einer erneuten Explantation des Knochendeckels bei Patienten nach autogener Knochendeckelreimplantation mit mehr als 2 Risikofaktoren ( $\mathrm{p}=0,073)$. Die registrierten internistischen Risikofaktoren waren: Diabetes mellitus, Gerinnungsstörung durch Thrombozytenaggregationshemmer oder Antikoagulanzien und das Vorliegen anderer Infektionen, insbesondere mit gegen Antibiotika multiresistenten Keimen. Hier fand sich mit $11(45,8 \%)$ von insgesamt 24 Fällen absolut und relativ der größte Anteil der Patienten mit lokaler Infektion des Knochendeckels. Patienten, die nach der Kraniektomie eine Nachblutung, einen Infarkt oder eine extrakranielle Infektion erlitten und zusätzlich mit ultrafrüher Kranioplastik behandelt wurden, hatten eine signifikant höhere Infektionsrate. Dies betraf 3 von den insgesamt 7 Fällen der Gruppe 1 (42,8\%). Ansonsten zeigten die Gruppen keinen signifikanten Unterschied in Bezug auf die Zahl von intrakraniellen Infektionen und Wundheilungsstörungen. Es fand sich auch keine statistische Signifikanz in der Inzidenz von Wundheilungsstörungen und Infektionen in Bezug auf Alter, Geschlecht und Ursache der Kraniektomie.

\section{Diskussion}

Bei der Planung der Kranioplastik wird die autologe Knochendeckelreimplantation aufgrund ihrer Kosteneffektivität, der natürlichen Histokompatibilität und Affinität in Verbindung mit den exzellenten kosmetischen Ergebnissen sowie der 

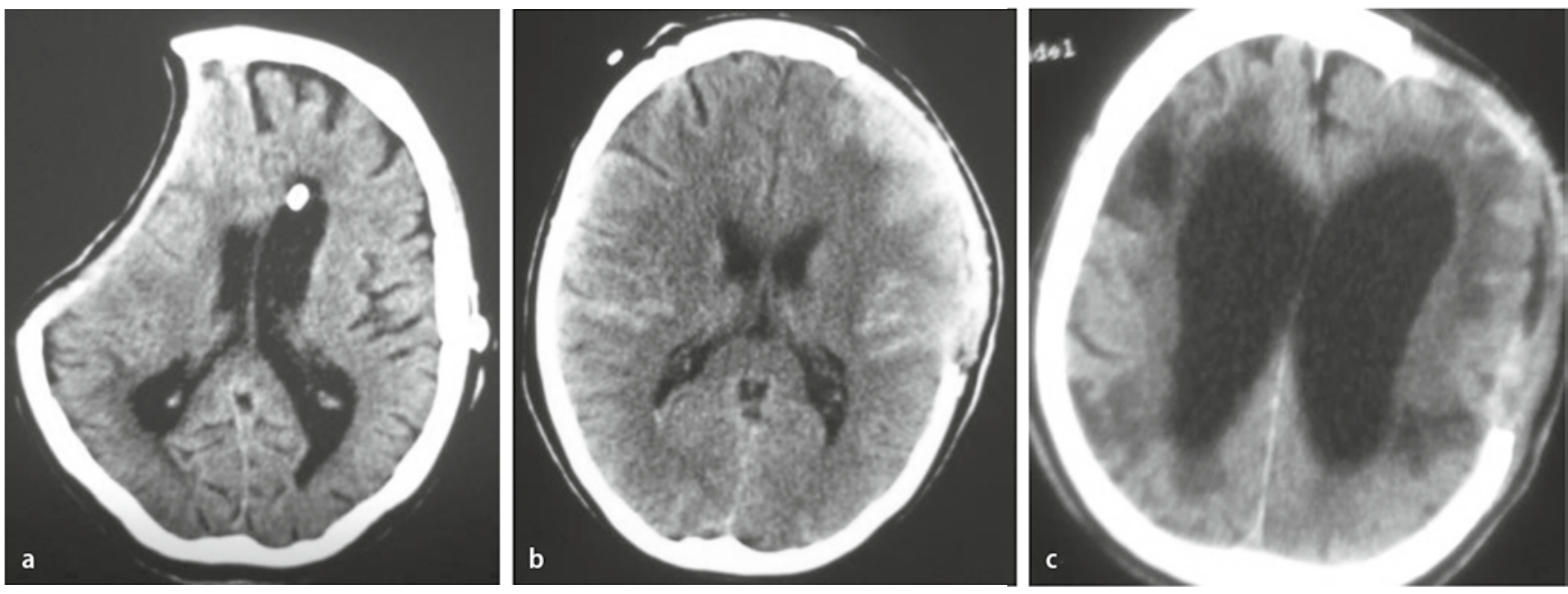

Abb. 4 A Veschiedene Kraniektomiedefekte nach CCT. a Typ 1: deutliche Exkavation des Kraniektomielappens. b Typ 2: Kraniektomielappen auf dem gleichen Niveau mit der Kalotte. c Typ 3: Kraniektomielappen über dem Kalottenniveau
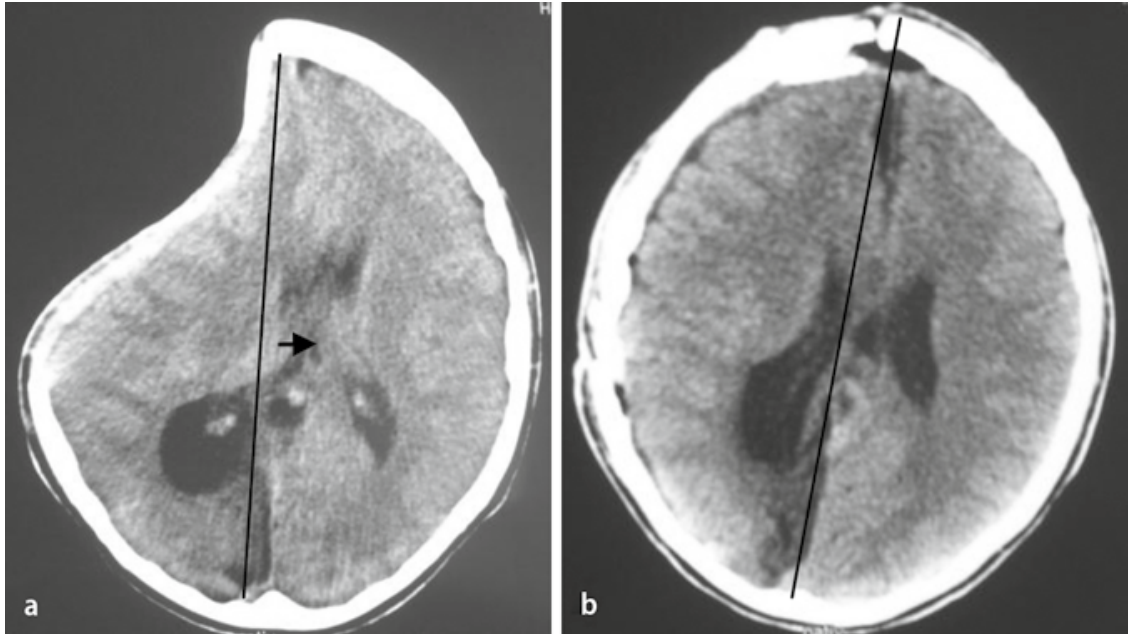

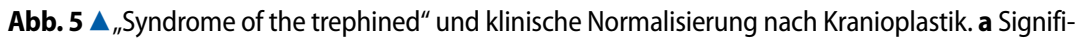
kante Mittellinienverlagerung bei einem Patient mit "syndrome of the trephined". b Normalisierung nach Kranioplastik mit einem PEEK-Implantat
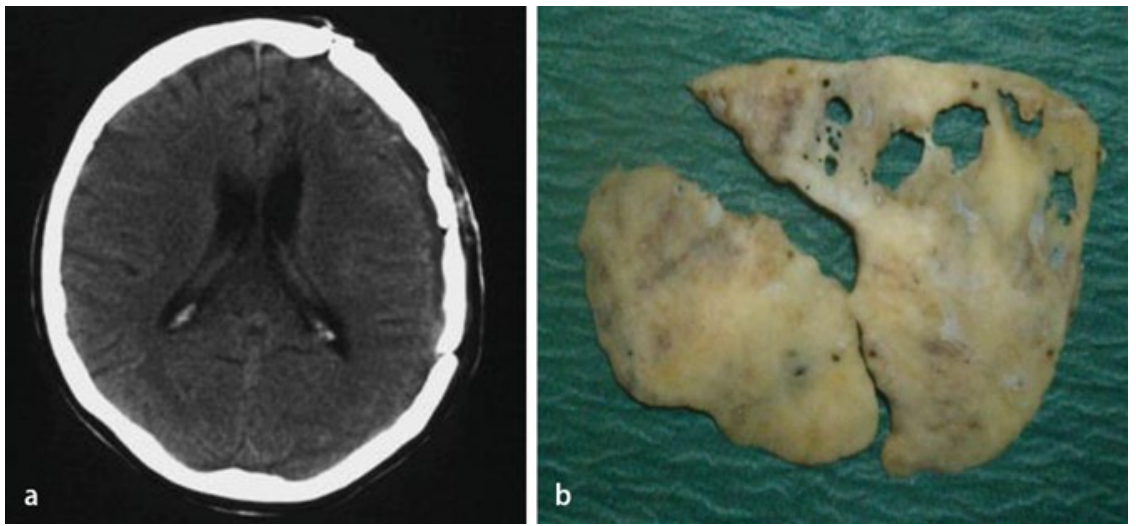

Abb. 6 \ Fallbeispiel einer Knochenresorption nach Knochendeckelreimplantation. a CCT-Kontrolle 2 Monate nach der Kranioplastik, sichtbare osteolytische Zonen im Bereich des Implantates im Sinne einer Knochenresorption. b Das autologe Implantat nach Explantation mit mehreren Resorptionsherden raschen Fusion des vitalen Knochengewebes [21, 38] als Mittel der 1 . Wahl betrachtet. Allerdings bleiben Nachteile bestehen wie: unvorhersehbare Resorption, begrenzte Verfügbarkeit und Kosten für die eventuelle Gabe von Wachstumsfaktoren $[3,16]$.

In dieser Arbeit erhielt die überwiegende Mehrzahl der Patienten (217 [90\%] von 242 Fällen) eine Kranioplastik durch Schädelknochenreimplantation des kryopreservierten Eigenknochens. Entscheidende Faktoren für die Bestimmung des Implantats sind: das Verhalten des Materials zu mechanischen, thermalen und elektrischen Reizen, die Verformbarkeit und Passgenauigkeit in den Knochendefekt, der Bedarf an biologischer Verträglichkeit, Anoxizität und das Fehlen von neoproliferativen und inflammatorischen Reaktionen der umliegenden Gewebe. Das ideale Knochensubstitut soll biokompatibel, osteokonduktiv und osteoinduktiv stabil sein.

Einige Autoren vertreten die Meinung, dass die Kranioplastik 3 bis 6 Monate nach der Kraniektomie durchgeführt werden kann [30]. Diese „konservative“ Haltung berücksichtigt zwar das Infektionsrisiko und die Zeitdauer bis zur Rückbildung der Primärschädigung, allerdings könnten die bereits erwähnten pathophysiologischen Veränderungen der Liquorzirkulation [8, 12, 29, 37], der zerebralen Perfusion [25, 27, 34] und des Metabolismus $[18,37]$ die Patienten zusätzlich benachteiligen. 
In unserer Studie ergab die Analyse der Patientengruppen, dass Patienten der Gruppe 1 (ultrafrühe Kranioplastik) ein besseres neurologisches Outcome hatten als Patienten der Gruppe 3 und 2. Die Beurteilung des neurologischen $\mathrm{Zu}$ stands vor der Kranioplastik war bei allen Gruppen vergleichbar. Die Patientengruppen nach Kranioplastikzeitpunkt weisen präoperativ keinen signifikanten demographischen oder klinischen Unterschied auf (• Tab. 1). Gemäß den Ergebnissen dieser Patientenserie kann die ultrafrühe Kranioplastik mittels Reimplantation des Eigenknochens als sicheres und hilfreiches Verfahren für die schnellere Rehabilitation und Besserung der neurologischen Funktion und Prognose bewertet werden. Zwischen Gruppe 1 und Gruppe 3 war eine statistische Signifikanz $(\mathrm{p}<0,05)$ zu verzeichnen, nicht dagegen zwischen Gruppe 1 und Gruppe 2. Die Ursachen hierfür könnten in einer geringen Fallzahl und in der kurzen Followup-Periode in der Mehrzahl der Fälle liegen sowie darin, dass $40 \%$ der Patienten der Gruppen 1 und 2 die Kranioplastik in den Wochen 5 bis 8 erhielten.

Trotz der Tatsache, dass in vorliegender Arbeit die Zeitspanne zwischen Kraniektomie und Kranioplastik zwischen 2 und 44 Wochen betrug, fand sich zwischen dem Zeitpunkt der Kranioplastik und der Resorption des Eigenknochens keine Korrelation. Mögliche Ursachen für die geringe Rate an Knochenresorptionen könnten sein:

- Die gewebsfreundliche Kryopreservation bei $-70^{\circ} \mathrm{C}$, ohne Sterilisation und ohne chemische Verarbeitung. Die Transplantate wurden nach dem gründlichen Waschen mit sterilem $\mathrm{NaCl}$ 0,9\% in Plastiktüten verpackt und in einem speziellen Kühlschrank gelagert. In manchen Studien wird die Methode zur Aufbewahrung des Knochens als die „übliche“ bezeichnet und nicht genau beschrieben. In anderen Studien werden niedrigere Temperaturen gewählt. Die strukturellen Proteine, die für die Revitalisierung des Knochendeckels notwendig sind, könnten dadurch denaturiert werden [13].

- Die starre Fixation des Knochendeckels mit der Umgebung mittels Ti- tanplättchen. DeLacure zeigte, dass der optimale stabile Kontakt der Knochenränder zur Osteokonduktion führt und eine Resorption verhindert [7]. Außerdem wird der Rand der Kraniektomie so frei präpariert, sodass der Kontakt zwischen Empfänger- und Spenderknochen maximiert wird, was die Aktivität der Osteoblasten in diesem Bereich fördert.

- Die geringe Zahl pädiatrischer Patienten (2 Patienten im Alter von 3 Jahren, 2 Patienten im Alter von 2 Jahren). Grant und Mitarbeiter stellten die Hypothese auf, dass die hohe Rate an Knochenresorptionen bei Kindern auf die Dünnheit des Schädelknochens zurückzuführen ist [13].

Die Kranioplastik hat nicht nur einen starken Einfluss auf die Dynamik der Liquorzirkulation, sondern auch auf die zerebrale Blutflussregulation, die zerebrovaskuläre Reservekapazität und den zerebralen Glukosemetabolismus [37]. Eine zusätzliche neurologische Besserung nach der Kranioplastik wird von vielen Autoren angegeben $[2,31,34]$. Agner und Mitarbeiter fanden bei ihren Patienten mittels detaillierten neurokognitiven Analysen eine signifikante Besserung in den kognitiven Funktionen nach der Kranioplastik eines großen Schädelknochendefektes. Diese neurokognitive Verbesserung wurde in direkten Zusammenhang mit dem CBF („cerebral blood flow“)-Anstieg gebracht [37].

Nach Kranioplastik wurde auch von mehreren Autoren über eine Besserung der Symptome des „syndrome of the trephined" und anderen neurokognitiven Funktionsstörungen berichtet [2, 8]. Andere Autoren beobachteten bei Syndrome-of-the-trephined-Patienten jedoch ein höheres Risiko für Komplikationen und ein schlechtes neurologisches Outcome, nämlich eine Hämatomentstehung im Bereich des Defektes und eine Dysfunktion der darunterliegenden kortikalen Strukturen [19].

In unsere Arbeit wurden 27 Patienten mit diesem Syndrom registriert. Das Vorkommen dieses Syndroms erklärt sich dadurch, dass alle diese Patienten einen großen supratentoriellen Kraniektomiedefekt hatten. Dies korreliert mit den Beob- achtungen anderer Autoren [8, 9]. Bei der Mehrzahl dieser Patienten in der Gruppe 1 und 2 kam es nach der Kranioplastik zur vollständigen Rückbildung dieser Symptome, wogegen es in der Gruppe 3 in den meisten Fällen nur zur Teilresolution oder Persistenz der Beschwerden kam. Eine mögliche Erklärung für das bessere Abschneiden der Patienten mit einer früh durchgeführten Kranioplastik könnten die früher eingesetzten, intensiven und risikogeminderten Rehabilitationsmaßnahmen darstellen.

Bei den Fällen ohne Reimplantation des Eigenknochens und in Anbetracht der großen Auswahl an Knochenersatzmaterialien bleiben viele offene Fragen bezüglich der Auswahl des Implantats [22]. Die Prozessierung von nicht ordnungsgemäß gelagerten Knochen mittels Tutoplast ${ }^{\circledast}$ ist ein zeitaufwendiges und kostenintensives Verfahren. Allerdings führt die präoperative Anfertigung von individuellen und passgenauen Implantaten durch den Einsatz der Computertechnik zu einer signifikanten Reduktion sowohl der Operationszeit als auch der Komplikationsrate bei Patienten mit großen Kraniektomiedefekten.

Alloplastische Materialien (Keramik, PMMA, Titan, PEEK) bleiben attraktive Alternativen. Eine postoperative Resorption ist zwar ausgeschlossen, allerdings könnte die Infektionsrate höher sein [13]. Andererseits war in dieser Studie die Operationszeit der Reimplantation des Eigenknochens signifikant kürzer als die Zeit für die Modellierung und Insertion einer PMMA-Plastik. Die benötigte Operationszeit bei CAD-CAM-Implantaten, wie PEEK und Bioverid, war vergleichbar. Titanimplantate waren besonders kostenintensiv und PMMA-Plastiken waren hinsichtlich des kosmetischen Ergebnisses unzuverlässig.

Der Einfluss der Kranioplastik auf der Liquorzirkulation ist schwer vorherzusagen und kann sich über mehrere Tage und Wochen erstrecken, sodass die Patienten im Verlauf auf Shuntpflichtigkeit bzw. Notwendigkeit der Druckänderung des implantierten Shuntventils kontrolliert werden sollten. Verschiedene Liquordruckuntersuchungen nach der Kranioplastik zeigen zwar die Normalisierung von vielen liquorhydrodynamischen Va- 
riablen [12], in einigen Fällen findet aber eine Steigerung des Liquorresorptionswiderstandes statt.

Dujovny und Mitarbeiter berichteten eine signifikante Veränderung des Liquorflusses sowie eine moderate Steigerung des venösen Rückflusses nach der Kranioplastik [10]. Häufig muss man bei Patienten mit großen Kraniektomiedefekten und gleichzeitig bestehender Störung der Liquorzirkulation im Sinne eines Hydrozephalus zunächst oder gleichzeitig ein ventrikuloperitoneales (VP)-Shuntsystem einbauen. In einem anderen $\mathrm{Re}$ port berichteten wir über die Ergebnisse der frühen kombinierten Kranioplastik und Implantation eines programmierbaren ventrikuloperitonealen Shunts bei 60 Patienten mit großen Defekten und Liquorzirkulationsstörungen [5]. Die frühe Kranioplastik mit Reimplantation des Eigenknochens kombiniert mit der Anlage eines ventrikuloperitonealen programmierbaren Shuntsystems erlaubte in solchen Fällen eine dynamische Anpassung an die Veränderungen des intrakraniellen Druckes und Liquorresorptionswiderstandes. Die kombinierte Therapie hat sich als sinnvoll erwiesen, insbesondere die Zahl der notwendigen Operationen (Kranioplastik, VP-Shunt-Anlage) und Komplikationen konnte somit reduziert werden [5]. Darüber hinaus gewährleistet sie auch deutliche Vorteile bezüglich des Outcomes der Patienten. Insofern sollten Patienten, die nach der Kranioplastik keine zusätzliche klinische Besserung oder sogar eine neurologische Verschlechterung erleben, routinemäßig auf eine zusätzliche Liquorzirkulationsstörung untersucht werden. Unsere aktuellen Ergebnisse bestätigen, dass das neurologische Outcome der Patienten in der Gruppe 1, die aufgrund Liquorzirkulationsstörung ein VP-Shunt als kombinierte Therapie in der gleichen Sitzung erhalten haben, im Vergleich zu den anderen Kranioplastikgruppen besser war. Somit stellt die frühe und ultrafrühe Kranioplastik bei Patienten mit konvexen, über Kalottenniveau prolabierten Kraniektomielappen aufgrund eines Hydrozephalus keine Kontraindikation mehr dar.

In dieser Studie traten in 24 Fällen (9,9\%) infektiöse Komplikationen auf. Als Infektion galt jede purulente oder nicht- purulente Infektion, die eine Entfernung des Implantats notwendig machte. Kleine Stichabszesse im Bereich der Hautfäden, die ohne Komplikationen verheilten, wurden ausgeschlossen. Die Ergebnisse dieser Arbeit korrelieren mit Ergebnissen anderer Autoren. Die Infektionsrate neurochirurgischer Operationen bei kontrolliert-randomisierten Studien beträgt ohne prophylaktische Beigaben von Antibiotika zwischen 4-12\% und mit Prophylaxe $0,3-3 \%$ [11]. In verschiedenen $\mathrm{Li}$ teraturstellen wird die Infektionsrate der Kranioplastik von mehreren Faktoren abhängig gemacht: Alter, Implantat, Lokalisation, Größe des Defektes und andere $[20,23]$. Nach Mazuno und Mitarbeiter war das Alter kein signifikanter Risikofaktor bei Erwachsenen [20]. Park und Mitarbeiter fanden ebenfalls keine statistisch signifikanten Unterschiede bei verschiedenen Altersgruppen und in Bezug auf das Geschlecht [23]. Unsere Ergebnisse korrelieren mit diesen Daten. Es wurde keine Korrelation zwischen Infektion, Alter, Geschlecht und Art des Implantats gefunden. Allerdings wurden andere begleitende Erkrankungen wie Diabetes mellitus, Gerinnungsstörung nach Thrombozytenaggregationshemmerund Antikoagulanzieneinnahmen sowie das Auftreten anderer Infektionen (insbesondere mit gegen Antibiotika multiresistenten Keimen) als infektionsassoziierte Risikofaktoren identifiziert. Insbesondere die Patienten mit autogener Knochendeckelreimplantation, die mehr als 2 dieser Risikofaktoren aufweisen, sind deutlich gefährdet. Hier findet sich mit 11 $(45,8 \%)$ von insgesamt 24 Fällen der absolut und relativ größte Anteil unter den Patienten mit lokaler Infektion des Knochendeckels. Eine Tendenz für Infektionen bestand auch bei Patienten, die sekundär andere Komplikation erlitten und einer ultrafrühen Kranioplastik unterzogen wurden. Patienten, die nach der Kraniektomie eine Nachblutung, Infarkt oder Infektion erlitten, hatten eine signifikant höhere Infektionsrate bei ultrafrüher Kranioplastik.

Eine retrospektive Analyse hat ihre Einschränkungen: Die begrenzten Eingabedaten schließen eine Regressionsanalyse aus, die variable zeitliche Länge des Follow-ups beeinflusst die Ergebnis- se zugunsten des kürzeren Follow-ups. Hinzu muss berücksichtigt werden, dass eine gewisse Verzerrung der Studienresultate aufgrund einer a priori Selektion von Patienten nicht ganz auszuschließen ist. Obwohl der Vergleich des präoperativen neurologischen Zustandes in den verschiedenen Gruppen keine wesentlichen Unterschiede zeigte, könnte ein Teil dieser eindeutigen Ergebnisse darauf beruhen, dass z. B. Patienten mit schwererem Krankheitsverlauf bei peristierendem Hirnödem oder ausgeprägterem Risikoprofil in die späte Kranioplastik-Gruppe vorselektiert wurden. Ein Selektionsbias konnte nicht identifiziert werden. Langzeitstudien mit größeren Patientenzahlen könnten dazu beitragen, noch ungeklärte Unterschiede zwischen Patientengruppen besser zu evaluieren.

\section{Fazit für die Praxis}

Die Ergebnisse dieser Arbeit bestätigen, dass die Kranioplastik nach einer supratentoriellen dekompressiven Kraniektomie mit Reimplantation des eigenen Schädelknochens zum frühesten möglichen Zeitpunkt (innerhalb den ersten 6 Wochen) ein sicheres und effektives Verfahren darstellt und bei ausgewählten Patienten sogar bessere Ergebnisse als die späte Kranioplastik haben kann. Das neurologische Outcome der Patienten in dieser Gruppe, bei denen ein kombiniertes Verfahren (z. B. Kranioplastik und VP-Shunt) durchgeführt wurde, ist auch vergleichbar mit dem der anderen Patientengruppen. Somit ist eine Kranioplastik bei Patienten mit konvexen, über Kalottenniveau prolabierten Kraniektomielappen aufgrund eines Hydrozephalus keine Kontraindikation.

Korrespondenzadresse
Neurochirurgische Klinik und
Ambulanz, Klinikum
Offenbach, Akademisches
Lehrkrankenhaus der JWG-
Universität Frankfurt am Main
Starkenburgring 66, 63069
Offenbach
neurosurgery@t-online.de


Interessenskonflikt. Der korrespondierende Autor gibt an, dass kein Interessenkonflikt besteht.

\section{Literatur}

1. Adamo MA, Deshaies EM (2008) Emergency decompressive craniectomy for fulminating infectious encephalitis. J Neurosurg 108:174-176

2. Agner C, Dujovny M, Gaviria M (2002) Neurocognitive assessment before and after cranioplasty. Acta Neurochir (Wien) 44:1033-1040

3. Arnaud E (2000) Advances in cranioplasty with osteoinductive biomaterials: summary of experimental studies and clinical prospects. Childs Nerv Syst 16:659-668

4. Bijlenga P, Zumofen D, Yilmaz H et al (2007) Orthostatic mesodiencephalic dysfunction after decompressive craniectomy. J Neurol Neurosurg Psychiatry 78:430-433

5. Carvi Y Nievas MN, Höllerhage HG (2006) Early combined cranioplasty and programmable shunt in patients with skull bone defects and CSF-circulation disorders. Neurol Res 28:139-144

6. Cooper DJ, Rosenfeld JV, Murray L et al (2008) Early decompressive craniectomy for patients with severe traumatic brain injury and refractory intracranial hypertension - a pilot randomized trial. J Crit Care 23:387-393

7. DeLacure MD (1994) Physiology of bone healing and bone grafts. Otolaryngol Clin North Am 27:859-874

8. Dujovny M, Agner C, Aviles A (1999) Syndrome of the trephined: theory and facts. Crit Rev Neurosurg 9:271-278

9. Dujovny M, Aviles A, Agner C et al (1997) Cranioplasty: cosmetic or therapeutic? Surg Neurol 47:238-241

10. Dujovny M, Fernandez P, Alperin N et al (1997) Post-cranioplasty cerebrospinal fluid hydrodynamic changes: magnetic resonance imaging quantitative analysis. Neurol Res 19:311-3116

11. Erman T, Demirhindi H, Göçer Al et al (2005) Risk factors for surgical site infections in neurosurgery patients with antibiotic prophylaxis. Surg Neurol 63:107-112

12. Fodstad H, Love JA, Ekstedt J et al (1984) Effect of cranioplasty on cerebrospinal fluid hydrodynamics in patients with the syndrome of the trephined. Acta Neurochir (Wien) 70:21-30

13. Grant GA, Jolley M, Ellenbogen RG et al (2004) Failure of autologous bone-assisted cranioplasty following decompressive craniectomy in children and adolescents. J Neurosurg 100:163-168

14. Hofmeijer J, Kappelle LJ, Algra A et al (2009) Surgical decompression for space-occupying cerebral infarction (the hemicraniectomy after middle cerebral artery infarction with life-threatening edema trial [HAMLET]): a multicentre, open, randomised trial. Lancet Neurol 8:326-333

15. Hutchinson PJ, Corteen E, Czosnyka M et al (2006) Decompressive craniectomy in traumatic brain injury: the randomized multicenter RESCUEicp study (www.RESCUEicp.com). Acta Neurochir 96:17-20

16. Iwama T, Yamada J, Imai S et al (2003) The use of frozen autogenous bone flaps in delayed cranioplasty revisited. Neurosurgery 52:591-596

17. Jüttler E, Schwab S, Schmiedek P et al (2007) Decompressive surgery for the treatment of malignant infarction of the middle cerebral artery (DESTINY): a randomized, controlled trial. Stroke 38:2518-2525

18. Yoshida K, Furuse M, Izawa A et al (1996) Dynamics of cerebral blood flow and metabolism in patients with cranioplasty as evaluated by $133 \mathrm{Xe} C \mathrm{CT}$ and 31P magnetic resonance spectroscopy. J Neurol Neurosurg Psychiatry 61:166-1671
19. Liao CC, Kao MC (2002) Cranioplasty for patients with severe depressed skull bone defect after cerebrospinal fluid shunting. J Clin Neurosci 9:553-555

20. Matsuno A, Tanaka H, Iwamuro H et al (2006) Analyses of the factors influencing bone graft infection after delayed cranioplasty. Acta Neurochir (Wien) 148:535-540

21. Moreira-Gonzalez A, Jackson IT, Miyawaki T et al (2003) Clinical outcome in cranioplasty: critical review in long-term follow-up. J Craniofac Surg 14:144-153

22. Park HK, Dujovny M, Agner C, Diaz FG (2001) Biomechanical properties of calvarium prosthesis. Neurol Res 23:267-276

23. Park JS, Lee KS, Shim JJ et al (2007) Large defekt may cause infectious compications in cranioplasty. J Korean Neurosurg Soc 42:89-91

24. Regel JP, Stolke D (2004) Dekompressive Kraniektomie aus neurochirurgischer Sicht. In: Moskopp D, Wassmann H (Hrsg) Neurochirurgie. Schattauer, Stuttgart S 231-238

25. Richaud J, Boetto S, Guell A, Lazorthes Y (1985) Effects of cranioplasty on neurological function and cerebral blood flow. Neurochirurgie 31183-188

26. Sanan A, Haines SJ (1997) Repairing holes in the head: a history of cranioplasty. Neurosurgery 40:588-603

27. Sakamoto S, Eguchi K, Kiura Y (2006) CT perfusion imaging in the syndrome of the sinking skin flap before and after cranioplasty. Clin Neurol Neurosurg 108:583-585

28. Sarov M, Guichard JP, Chibarro S (2010) DECIMAL investigators. Sinking skin flap syndrome and paradoxical herniation after hemicraniectomy for malignant hemispheric infarction. Stroke 41:560 562

29. Schiffer J, Gur R, Nisim U, Pollak L (1997) Symptomatic patients after craniectomy. Surg Neurol 47:231-237

30. Schimidek H (2000) Operative Neurosurgical Technique: Cranioplasty: Indications, Technique and Prognosis. 4. Aufl. Elsevier Science, Singapore

31. Segal DH, Oppenheim JS, Murovic JA (1994) Neurological recovery after cranioplasty. Neurosurgery 34:729-731

32. Smith ER, Carter BS, Ogilvy CS (2002) Proposed use of prophylactic decompressive craniectomy in poor-grade aneurysmal subarachnoid hemorrhage patients presenting with associated large sylvian hematomas. Neurosurgery 51:117-124

33. Steiger HJ, Reulen (1999) Manual Neurochirurgie. Ecomed Verlagsgesellschaft, Landsberg, S 299302

34. Suzuki N, Suzuki S, Iwabuchi T (1993) Neurological improvement after cranioplasty. Analysis by dynamic CT scan. Acta Neurochir (Wien) 122:49-53

35. Unterberg A, Kirkpatrick PJ (2006) Decompressive craniectomy in traumatic brain injury: the randomized multicenter RESCUEicp study (www.RESCUEicp.com). Acta Neurochir 96:17-20

36. Vahedi K, Hofmeijer J, Juettler E (2007) Early decompressive surgery in malignant infarction of the middle cerebral artery: a pooled analysis of three randomised controlled trials. Lancet Neurol 6:215222

37. Winkler PA, Stummer W, Linke R et al (2000) Influence of cranioplasty on postural blood flow regulation, cerebrovascular reserve capacity, and cerebral glucose metabolism. J Neurosurg 93:53-61

38. Zingale A, Albanese V (2003) Cryopreservation of autogeneous bone flap in cranial surgical practice: what is the future? A grade B and evidence level 4 meta-analytic study. J Neurosurg Sci 47:137-139

\section{Gefahr durch Hirnblutungen erkennen, bevor sie entstehen}

Etwa 2-3\% aller Erwachsenen entwickeln im Laufe ihres Lebens ein Hirnaneurysma. In Deutschland sind 1,5 bis 2 Millionen Menschen betroffen. Ein Aneurysma birgt stets die Gefahr zu reißen und eine lebensbedrohliche Blutung zu verursachen. Das Risiko einer Ruptur besteht bei etwa $5 \%$ in einem Zeitraum von 5 Jahren. Reißt das Aneurysma, kommt es zu einer Subarachnoidalblutung. Aneurysmen treten oft familiär gehäuft auf. Für Verwandte ersten Grades eines Betroffenen ist das Risiko, selbst ein Aneurysma zu tragen, 3- bis 7-fach erhöht. Bei Menschen, in deren nahen Verwandtenkreis bereits Aneurysmen aufgetreten sind, kann eine Abklärung sinnvoll sein. Im Falle eines Funds besteht die Möglichkeit einer Behandlung durch die endovaskuläre Therapie. Dabei werden platinbeschichtete Spiralen in das Blutgefäß eingeführt und die Aussackung damit teilweise ausgefüllt. Dies verhindert die weitere Blutzirkulation im Aneurysma, was das Risiko einer Subarachnoidalblutung senkt. Obwohl das Behandlungsrisiko der endovaskulären Therapie in den letzten Jahren deutlich gesenkt wurde, treten bei etwa $5 \%$ der Patienten Komplikationen auf. Ähnlich risikobehaftet ist auch der chirurgische Eingriff zur Ausschaltung eines Aneurysmas. Aus diesen Gründen müssen Arzt und Patient bereits vor dem Screening genau abwägen, was der mögliche Befund für die Lebensqualität des Patienten bedeutet und welche Risiken eine Behandlung birgt. Sofern das Behandlungsrisiko gering ist und keine Gründe gegen eine Operation sprechen, rät die DGNR zur Behandlung in spezialisierten Zentren. Dies vor allem auch, um die Lebensqualität der Patienten zu verbessern, die von der schlummernde Gefahr in ihrem Kopf wissen.

Quelle: Deutsche Gesellschaft für Neuroradiologie (DGNR), www.neuroradiologie.de 